\title{
LOG-CONCAVITY OF COMPLEXITY ONE HAMILTONIAN TORUS ACTIONS
}

\author{
YUNHYUNG CHO AND MIN KYU KIM
}

\begin{abstract}
Let $(M, \omega)$ be a closed $2 n$-dimensional symplectic manifold equipped with a Hamiltonian $T^{n-1}$-action. Then Atiyah-Guillemin-Sternberg convexity theorem implies that the image of the moment map is an $(n-1)$-dimensional convex polytope. In this paper, we show that the density function of the Duistermaat-Heckman measure is logconcave on the image of the moment map.
\end{abstract}

\section{INTRODUCTION}

In statistic physics, the relation $S(E)=k \log W(E)$ is called Boltzmann's principle where $W$ is the number of states with given values of macroscopic parameters $E$ (like energy, temperature, ..), $k$ is the Boltzmann's constant, and $S$ is the entropy of the system which measures the degree of disorder in the system. For the additive values $E$, it is wellknown the entropy is always concave function. (See O1 for more detail). In symplectic setting, consider a Hamiltonian $G$-manifold $(M, \omega)$ with the moment map $\mu: M \rightarrow \mathfrak{g}^{*}$. The Liouville measure $m_{L}$ is defined by

$$
m_{L}(U):=\int_{U} \frac{\omega^{n}}{n !}
$$

for any open set $U \subset M$. Then the push-forward measure $m_{\mathrm{DH}}:=\mu_{*} m_{L}$, called the Duistermaat-Heckman measure, can be regarded as a measure on $\mathfrak{g}^{*}$ such that for any Borel subset $B \subset \mathfrak{g}^{*}, m_{\mathrm{DH}}(B)=\int_{\mu^{-1}(B)} \frac{\omega^{n}}{n !}$ tells us that how many states of our system have momenta in $B$. By the Duistermaat-Heckman theorem [DH], $m_{\mathrm{DH}}$ can be expressed in terms of the density function $\mathrm{DH}(\xi)$ with respect to the Lebesque measure on $\mathfrak{g}^{*}$. Therefore the concavity of the entropy of given Hamiltonian system on $(M, \omega)$ can be interpreted as the log-concavity of $\mathrm{DH}(\xi)$ on the image of $\mu$. A. Okounkov [O2] proved that the density function of the Duistermaat-Heckman measure is log-concave on the image of the moment map for the maximal torus action when $(M, \omega)$ is the co-adjoint orbit of some classical Lie groups. In $[\mathrm{Gr}], \mathrm{W}$. Graham showed the log-concavity of the density function of the Duistermaat-Heckman measure also holds for any Kähler manifold admitting a holomorphic Hamiltonian torus action. V. Ginzberg and A. Knudsen conjectured independently that the log-concavity holds for any Hamiltonian $G$-manifolds, but it turns out to be false in general by Y. Karshon [K1]. Further related works can be found in [L] and [C].

Date: April 27, 2022. 
As noted in [K1] and [Gr], log-concavity holds for Hamiltonian toric (i.e. complexity zero) actions, and Y. Lin dealt with log-concavity of complexity two Hamiltonian torus actions in $[\mathrm{L}$. But, there is no result on log-concavity of complexity one Hamiltonian torus actions. This is why we rstrict our interest to complexity one. From now on, we assume that $(M, \omega)$ is a $2 n$-dimensional closed symplectic manifold with an effective Hamiltonian $T^{n-1}$-action. Let $\mu: M \rightarrow \mathfrak{t}^{*}$ be the corresponding moment map where $\mathfrak{t}^{*}$ is a dual of the Lie algebra of $T^{n-1}$. By Atiyah-Guillemin-Sternberg convexity theorem, the image of the moment map $\mu(M)$ is an $(n-1)$-dimensional convex polytope in $\mathfrak{t}^{*}$. By the DuistermaatHeckman theorem [DH], we have

$$
m_{\mathrm{DH}}=\mathrm{DH}(\xi) d \xi
$$

where $d \xi$ is the Lebesque measure on $\mathfrak{t}^{*} \cong \mathbb{R}^{n-1}$ and $\mathrm{DH}(\xi)$ is a continuous piecewise polynomial function of degree less than 2 on $\mathfrak{t}^{*}$. Our main theorem is as follow.

Theorem 1.1. Let $(M, \omega)$ be a $2 n$-dimensional closed symplectic manifold equipped with a Hamiltonian $T^{n-1}$-action with the moment map $\mu: M \rightarrow \mathfrak{t}^{*}$. Then the density function of the Duistermaat-Heckman measure is log-concave on $\mu(M)$.

\section{Proof of the theorem 1.1}

Let $(M, \omega)$ be a $2 n$-dimensional closed symplectic manifold. Let $(n-1)$-dimensional torus $T$ acts on $(M, \omega)$ in Hamiltonian fashion. Denote by $\mathfrak{t}$ the Lie algebra of $T$. For a moment map $\mu: M \rightarrow \mathfrak{t}^{*}$ of the $T$-action, define the Duistermaat-Heckman function $\mathrm{DH}: \mathfrak{t}^{*} \rightarrow \mathbb{R}$ as

$$
\mathrm{DH}(\xi)=\int_{M_{\xi}} \omega_{\xi}
$$

where $M_{\xi}$ is the reduced space $\mu^{-1}(\xi) / T$ and $\omega_{\xi}$ is the corresponding reduced symplectic form on $M_{\xi}$.

Now, we define the x-ray of our action. Let $T_{1}, \cdots, T_{N}$ be the subgroups of $T^{n-1}$ which occur as stabilizers of points in $M^{2 n}$. Let $M_{i}$ be the set of points whose stabilizers are $T_{i}$. By relabeling, we can assume that $M_{i}$ 's are connected and the stabilizer of points in $M_{i}$ is $T_{i}$. Then, $M^{2 n}$ is a disjoint union of $M_{i}$ 's. Also, it is well known that $M_{i}$ is open dense in its closure and the closure is just a component of the fixed set $M^{T_{i}}$. Let $\mathfrak{M}$ be the set of $M_{i}$ 's. Then, the $x$-ray of $\left(M^{2 n}, \omega, \mu\right)$ is defined as the set of $\mu\left(\overline{M_{i}}\right)$ 's. Here, we recall a basic lemma.

Lemma 2.1. [GS, Theorem 3.6] Let $\mathfrak{h}$ be the Lie algebra of $T_{i}$. Then $\mu\left(M_{i}\right)$ is locally of the form $x+\mathfrak{h}^{\perp}$ for some $x \in \mathfrak{t}^{*}$.

By this lemma, $\operatorname{dim}_{\mathbb{R}} \mu\left(M_{i}\right)=m$ for $(n-1-m)$-dimensional $T_{i}$. Each image $\mu\left(\overline{M_{i}}\right)$ (resp. $\left.\mu\left(M_{i}\right)\right)$ is called an $m$-face (resp. an open $m$-face) of the x-ray if $T_{i}$ is $(n-1-m)$ dimensional. Our interest is mainly in open $(n-2)$-faces of the x-ray, i.e. codimension one in $\mathfrak{t}^{*}$. Figure 2.1 is an example of x-ray with $n=3$ where thick lines are $(n-2)$-faces. Now, we can prove the main theorem. 


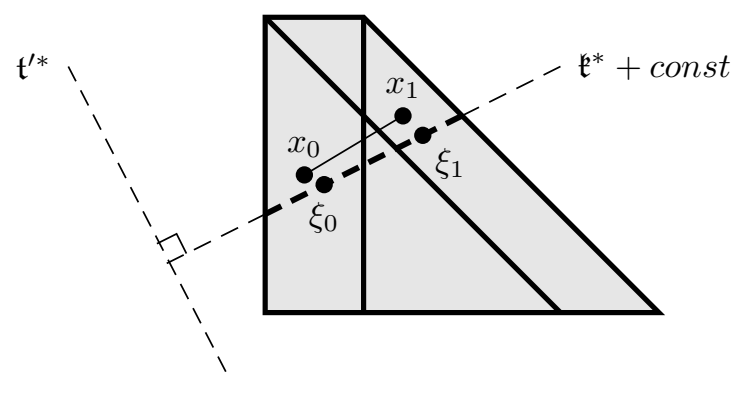

Figure 2.1. Proof of Theorem 1.1

Proof of Theorem [1.1. When $n=2$, we obtain a proof by [K2, Lemma 2.19]. So, we assume $n \geq 3$. Pick arbitrary two points $x_{0}, x_{1}$ in the image of $\mu$. We should show that

$$
t \log \left(\mathrm{DH}\left(x_{1}\right)\right)+(1-t) \log \left(\mathrm{DH}\left(x_{0}\right)\right) \leq \log \left(\mathrm{DH}\left(t x_{1}+(1-t) x_{0}\right)\right)
$$

for each $t \in[0,1]$. Put $x_{t}=t x_{1}+(1-t) x_{0}$.

Let us fix a decomposition $T=S^{1} \times \cdots \times S^{1}$. By the decomposition, we identify $\mathfrak{t}$ with $\mathbb{R}^{n-1}$, and $\mathfrak{t}$ carries the usual Riemannian metric $\langle,\rangle_{0}$ which is a bi-invariant metric. This metric gives the isomorphism

$$
\iota: \mathfrak{t} \rightarrow \mathfrak{t}^{*}, X \mapsto\langle\cdot, X\rangle_{0} .
$$

For a small $\epsilon>0$, pick two regular values $\xi_{i}$ in the ball $B\left(x_{i}, \epsilon\right)$ for $i=0,1$ which satisfy the following two conditions:

i. $\xi_{1}-\xi_{0} \in \iota\left(\mathbb{Q}^{n-1}\right)$,

ii. the line $L$ containing $\xi_{0}, \xi_{1}$ in $\mathfrak{t}^{*}$ meets each open $m$-face transversely for $m=$ $1, \cdots, n-2$.

Transversality guarantees that the line does not meet any open $m$-face for $m \leq n-3$. Put

$$
\xi_{t}=t \xi_{1}+(1-t) \xi_{0} \text { and } X=\iota^{-1}\left(\xi_{1}-\xi_{0}\right) .
$$

Let $\mathfrak{k} \subset \mathfrak{t}$ be the one-dimensional subalgebra spanned by $X$. By i., $\mathfrak{k}$ becomes a Lie algebra of a circle subgroup of $T$, call it $K$. Let $\mathfrak{t}^{\prime}$ be the orthogonal complement of $\mathfrak{k}$ in $\mathfrak{t}$. Again by i., $\mathfrak{t}^{\prime}$ becomes a Lie subgroup of a $(n-2)$-dimensional subtorus of $T$, call it $T^{\prime}$. Let

$$
p: \mathfrak{t}^{*} \rightarrow \mathfrak{t}^{*}=\iota\left(\mathfrak{t}^{\prime}\right)
$$

be the orthogonal projection along $\mathfrak{k}^{*}=\iota\left(\mathfrak{k}^{\prime}\right)$. If we put $\mu^{\prime}=p \circ \mu$, then $\mu^{\prime}: M \rightarrow \mathfrak{t}^{\prime *}$ is a moment map of the restriced $T^{\prime}$-action on $M$. Put $\xi^{\prime}=p\left(\xi_{t}\right)$ for $t \in[0,1]$.

We would show that $\xi^{\prime}$ is a regular value of $\mu^{\prime}$. For this, we show that each point $x \in \mu^{\prime-1}\left(\xi^{\prime}\right)$ is a regular point of $\mu^{\prime}$. By ii. and Lemma 2.1, stabilizer $T_{x}$ is finite or onedimensional. If $T_{x}$ is finite, then $x$ is a regular point of $\mu$ so that it is also a regular point of $\mu^{\prime}$. If $T_{x}$ is one-dimensional, then $\mu(x)$ is a point of an open $(n-2)$-face $\mu\left(M_{i}\right)$ such that $x \in M_{i}$. Let $\mathfrak{h}$ be the Lie algebra of $T_{i}=T_{x}$. By Lemma 2.1, $p\left(d \mu\left(T_{x} M_{i}\right)\right)=p\left(\mathfrak{h}^{\perp}\right)$, and the kernel $\mathfrak{k}$ of $p$ is not contained in $\mathfrak{h}^{\perp}$ by transversality. So, $p\left(\mathfrak{h}^{\perp}\right)$ is the whole $\mathfrak{t}^{\prime *}$ because 
$\operatorname{dim} \mathfrak{h}^{\perp}=\operatorname{dim} \mathfrak{t}^{\prime *}$, and this means that $x$ is a regular point of $\mu^{\prime}$. Therefore, we have shown that $\xi^{\prime}$ is a regular value of $\mu^{\prime}$.

Since $\xi^{\prime}$ is a regular value, the preimage $\mu^{\prime-1}\left(\xi^{\prime}\right)$ is a manifold and $T^{\prime}$ acts almost freely on it, i.e. stabilizers are finite. So, if we denote by $M_{\xi^{\prime}}$ the symplectic reduction $\mu^{\prime-1}\left(\xi^{\prime}\right) / T^{\prime}$, then it becomes a symplectic orbifold carrying the induced symplectic $T / T^{\prime}$-action. We can observe that the image of $\mu^{\prime-1}\left(\xi^{\prime}\right)$ through $\mu$ is the thick dashed line in Figure 2.1, Since $K /\left(K \cap T^{\prime}\right) \cong T / T^{\prime}$, we will regard $K /\left(K \cap T^{\prime}\right)$ and $\mathfrak{k}$ as $T / T^{\prime}$ and its Lie algebra, respectively. The map $\mu_{X}:=\langle\mu, X\rangle$ induces a map on $M_{\xi^{\prime}}$ by $T$-invariance of $\mu$, call it just $\mu_{X}$ where $\langle\rangle:, \mathfrak{t}^{*} \times \mathfrak{t} \rightarrow \mathbb{R}$ is the evaluation pairing. Then, we can observe that $\mu_{X}$ is a Hamiltonian of the $K /\left(K \cap T^{\prime}\right)$-action on $M_{\xi^{\prime}}$, and that $M_{\xi_{t}}$ is symplectomorphic to the symplectic reduction of $M_{\xi^{\prime}}$ at the regular value $\left\langle\xi_{t}, X\right\rangle$ with respect to $\mu_{X}$. If we denote by $\mathrm{DH}_{X}$ the Duistermaat-Heckman function of $\mu_{X}: M_{\xi^{\prime}} \rightarrow \mathbb{R}$, then we have $\mathrm{DH}\left(\xi_{t}\right)=\mathrm{DH}_{X}\left(\left\langle\xi_{t}, X\right\rangle\right)$ for $t \in[0,1]$. Since $M_{\xi^{\prime}}$ is a four-dimensional symplectic orbifold with Hamiltonian circle action, $\mathrm{DH}_{X}$ is log-concave by Lemma 2.2 below. Since $x_{t}$ and $\xi_{t}$ are sufficiently close and DH is continuous by [DH], we can show (2.1) by log-concavity of $\mathrm{DH}_{X}$.

Lemma 2.2. Let $(N, \sigma)$ be a closed four dimensional Hamiltonian $S^{1}$-orbifold. Then the density function of the Duistermaat-Heckman measure is log-concave.

Proof. Let $\phi: N \rightarrow \mathbb{R}$ be a moment map. Then the density function $\mathrm{DH}: \operatorname{Im} \phi \rightarrow \mathbb{R}_{\geq 0}$ of the Duistermaat-Heckman measure is given by

$$
\mathrm{DH}(t)=\int_{N_{t}} \sigma_{t}
$$

for any regular value $t \in \operatorname{Im} \phi$. Let $(a, b) \subset \operatorname{Im} \phi$ be an open interval consisting of regular values of $\phi$ and fix $t_{0} \in(a, b)$. By the Duistermaat-Heckman theorem $[\mathrm{DH}],\left[\sigma_{t}\right]-\left[\sigma_{t_{0}}\right]=$ $-e\left(t-t_{0}\right)$ for any $t \in(a, b)$, where $e$ is the Euler class of the $S^{1}$-fibration $\phi^{-1}\left(t_{0}\right) \rightarrow$ $\phi^{-1}\left(t_{0}\right) / S^{1}$. Therefore

$$
D H^{\prime}(t)=-\int_{N_{t}} e
$$

and

$$
D H^{\prime \prime}(t)=0
$$

for any $t \in(a, b)$. Note that $\mathrm{DH}(t)$ is log-concave on $(a, b)$ if and only if it satisfies $\mathrm{DH}(t) \cdot \mathrm{DH}^{\prime \prime}(t)-\mathrm{DH}^{\prime}(t)^{2} \leq 0$ for all $t \in(a, b)$. Hence $\mathrm{DH}(t)$ is log-concave on any open intervals consisting of regular values.

Let $c$ be any interior critical value of $\phi$ in $\operatorname{Im} \phi$. Then it is enough to show that the jump in the derivative of $(\log \mathrm{DH})^{\prime}$ is negative at $c$. First, we will show that the jump of the value $\mathrm{DH}^{\prime}(t)=-\int_{N_{t}} e$ is negative at $c$. Choose a small $\epsilon>0$ such that $(c-\epsilon, c+\epsilon)$ does not contain a critical value except for $c$. Let $N_{c}$ be a symplectic cut of $\phi^{-1}[c-\epsilon, c+\epsilon]$ along the extremum so that $N_{c}$ becomes a closed Hamiltonian $S^{1}$-orbifold whose maximum is the reduced space $M_{c+\epsilon}$ and the minimum is $N_{c-\epsilon}$. Using the Atiyah-Bott-Berline-Vergne localization formula for orbifolds [M], we have 


$$
0=\int_{N_{c}} 1=\sum_{p \in N^{S^{1} \cap \phi^{-1}(c)}} \frac{1}{d_{p}} \frac{1}{p_{1} p_{2} \lambda^{2}}+\int_{M_{c-\epsilon}} \frac{1}{\lambda+e_{-}}+\int_{N_{c+\epsilon}} \frac{1}{-\lambda-e_{+}}
$$

which is equivalent to

$$
0=\sum_{p \in N^{S^{1} \cap \phi^{-1}}(c)} \frac{1}{p_{1} p_{2}}=\int_{N_{c-\epsilon}} e_{-}-\int_{N_{c+\epsilon}} e_{+},
$$

where $d_{p}$ is the order of the local group of $p, p_{1}$ and $p_{2}$ are the weights of the tangential $S^{1}$ representation on $T_{p} N$, and $e_{-}$( $e_{+}$respectively) is the Euler class of $\phi^{-1}(c-\epsilon)\left(\phi^{-1}(c+\epsilon)\right.$ respectively). Since $c$ is in the interior of $\operatorname{Im} \phi$, we have $p_{1} p_{2}<0$ for any $p \in N^{S^{1}} \cap \phi^{-1}(c)$. Hence the jump of $\mathrm{DH}^{\prime}(t)=-\int_{N_{t}} e$ is negative at $c$, which implies that the jump of $\log \mathrm{DH}(t)^{\prime}=\frac{\mathrm{DH}^{\prime}(t)}{\mathrm{DH}(t)}$ is negative at $c$ (by continuity of $\mathrm{DH}(t)$ ). It finishes the proof.

\section{REFERENCES}

[C] Y. Cho, The log-concavity conjecture on semifree symplectic $S^{1}$-manifolds with isolated fixed points, arXiv:1103.2998

[DH] J. J. Duistermaat and G. J. Heckman, On the variation in the cohomology of the symplectic form of the reduced phase space, Invent. Math. 69 (1982), 259-268.

[Gr] W. Graham, Logarithmic convexity of push-forward measures, Invent. Math. 123 (1996), 315-322.

[GS] V. Guillemin and S. Sternberg, Convexity property of the moment mapping, Invent. Math. 67 (1982), 491-513.

[K1] Y. Karshon, Example of a non-log-concave Duistermaat-Heckman measure, Mathematical Research Letters 3 (1996), no. 4, 537-540.

[K2] Y. Karshon, Periodic Hamiltonian flows on four dimensional manifolds, Mem. Amer. Math. Soc. 141 (1999), no. 672.

[L] Y. Lin, The log-concavity conjecture for the Duistermaat-Heckman measure revisited, Int. Math. Res. Not. (2008), no. 10, Art. ID rnn027, 19 pp.

[M] E. Meinrenken, Symplectic surgery and the Spin ${ }^{c}$-Dirac operators, Advances in Mathematics, 134 (1998), 240-277.

[O1] A. Okounkov. Why would multiplicities be log-concave?, The Orbit Method in Geometry and Physics Boston, MA : Birkhauser Boston; (2003), Progress in Mathematics, 213 (Marseille, 2000), 329-347.

[O2] A. Okounkov, Log-concavity of multiplicities with application to characters of $U(\infty)$, Advanced in Mathematics. 127 (1997), 258-282.

School of Mathematics, Korea Institute for Advanced Study, 87 Hoegiro, DongdaemunGu, SeOul, 130-722, Republic of KoreA

E-mail address: yhcho@kias.re.kr

Department of Mathematics Education, Gyeongin National University of Education, SAn 59-12, Gyesan-dong, Gyeyang-Gu, Incheon, 407-753, Republic of Korea

E-mail address: mkkim@kias.re.kr 